\title{
Wrapping PDF Documents Exploiting Uncertain Knowledge
}

\author{
Sergio Flesca ${ }^{1}$, Salvatore Garruzzo ${ }^{2}$, Elio Masciari ${ }^{3}$, and Andrea Tagarelli ${ }^{1}$ \\ 1 DEIS, University of Calabria \\ fflesca, tagarelli\}@deis.unical.it \\ 2 DIMET, University of Reggio Calabria \\ salvatore.garruzzo@unirc.it \\ 3 ICAR-CNR - Institute of Italian National Research Council \\ masciari@icar.cnr.it
}

\begin{abstract}
The PDF format represents the de facto standard for printoriented documents. In this paper we address the problem of wrapping PDF documents, which raises new challenges in the information extraction field. The proposal is based on a novel bottom-up wrapping approach to extract information tokens and integrate them into groups related according to the logical structure of a document. A PDF wrapper is defined by specifying a set of group type definitions which impose a target structure to token groups containing the required information. Due to the intrinsic uncertainty on the structure and presentation of PDF documents, we devise constraints on token groupings as fuzzy logic conditions. We define a formal semantics for PDF wrappers and propose an algorithm for wrapper evaluation working in polynomial time with respect to the size of a $\mathrm{PDF}$ document.
\end{abstract}

\section{Introduction}

In the context of Information Extraction, wrapping is the process of extracting data containing information pertinent to a specific application domain, and organizing such data into a machine-readable format. Traditional wrapping refers to the Web environment; Web wrapping systems (e.g. [1, 2, 3, 4, 5, 6]) exploit markup tags in HTML pages to generate delimiter-based extraction rules 7 .

However, HTML and XML are not the only formats to spread and exchange textual information for the purpose of making it accessible to companies and private users. A further and related kind of textual document refers to print-oriented formats, whose Acrobat PDF [8] is the de facto standard. A PDF document is described by a PDF content stream, which contains a sequence of graphical and textual objects located at precise positions inside the document pages. Such intrinsic print-oriented nature of PDF documents arises many issues that make the information extraction task particularly difficult.

As a motivating application scenario, consider PDF documents describing company balance sheets, like that of Fig. 1. Automatically extracting information from balance sheets is extremely useful to build data warehouses of financial data. Balance sheets are statements of the total assets and liabilities of 


\begin{tabular}{|c|c|c|c|}
\hline \multicolumn{4}{|c|}{$\begin{array}{l}\text { Balance Sheet for XYZ Manufacturing Company } \\
\text { as of Dec } 31200\end{array}$} \\
\hline \multicolumn{4}{|l|}{\begin{tabular}{|l} 
(All Figures in USD) \\
\end{tabular}} \\
\hline Assets & 200 & Liabilities and Owners' Equity & 200 \\
\hline Current Assets & $5,000,000$ & Current Liabilities & \\
\hline Cash & 500,000 & Accounts Payable & $4,000,000$ \\
\hline T-Bills & $1,000,000$ & Dividend Payable & $2,000,000$ \\
\hline Accounts Receivable & $7,000,000$ & Taxes Payable & $3,000,000$ \\
\hline Total Current Assets & $13,500,000$ & Total Current Liabilities & $9,000,000$ \\
\hline inventory & & Long-term Liabilities & \\
\hline Raw Materials & 825,000 & Long-term Bank Loan & $5,000,000$ \\
\hline WIP & 750,000 & Total Liabilities & $14,000,000$ \\
\hline Finished Goods & $1,200,000$ & Owners' Equity & \\
\hline \begin{tabular}{|l|} 
Total Inventory \\
\end{tabular} & $2,775,000$ & Capital & $20,000,000$ \\
\hline Long-term assets & & Retained Earnings & $28,275,000$ \\
\hline Land & $30,000,000$ & Total Net Worth & $48,275,000$ \\
\hline \multicolumn{4}{|c|}{$20,000,000$} \\
\hline \multicolumn{4}{|c|}{\begin{tabular}{|l|l|} 
Depreciation (machinery) & $-5,000,000$ \\
\end{tabular}} \\
\hline \multicolumn{4}{|l|}{ intangible Assets } \\
\hline Patents & $1,000,000$ & & \\
\hline Total Long-term Assets & $46,000,000$ & & \\
\hline Total Assets & $62,275,000$ & Total Liabilities + Net Worth & $62,275,000$ \\
\hline
\end{tabular}

Fig. 1. Excerpt of a sample balance sheet

an organization, at a particular date, and are usually available as PDF documents. Each company may encode balance data using different presentation styles (e.g. two-column or four-column layout, different instructions for text formatting etc.). The subjectivity in the layout structure that characterize even thematically similar balance sheets leads to "uncertainty" in the specification of the syntactic extraction rules.

To date, the problem of wrapping PDF documents has not been studied at all, in spite of its applicability to a wide variety of scenarios. No existing wrapper generation system is designed for print-oriented documents. At a first sight, it is evident that while most information extraction approaches can be extended to deal with the characteristics of the PDF format, the main hypothesis enabling most wrapping approaches is lacking: even if documents are yet automatically produced using data coming from company databases, each company can use a different program to encode data in a document and, consequently, the resulting layouts can be different.

In this paper we address the problem of extracting information from PDF documents by focusing on their spatial and content features. We propose a novel bottom-up wrapping approach which considers the complex schema of the information to be extracted and exploits logical fuzzy rule-based conditions on the extracted information. The combined use of bottom-up extraction and fuzzy conditions enables effectively handling uncertainty on the comprehension of the layout structure of PDF documents.

Section 2 introduces basic notions for dealing with PDF documents as sets of spatial tokens, and provides background on spatial relations and fuzzy set theory. Section 3 describes a novel framework for wrapping PDF documents and a semantics for PDF wrappers. Section 4 addresses the PDF wrapper evaluation issue and describes an algorithm for extracting a maximal token group from a source PDF 
document, which works in polynomial time with respect to the size (number of tokens) of the source document. Section 5 contains concluding remarks.

\section{Preliminaries}

\section{$2.1 \quad$ Fuzzy Sets}

The sharp nature of classic set theory may often lead to scenarios in which the exact assignment of an object to a set is hardly obtained or unfeasible. Fuzzy set theory 9 takes into account the uncertainty due to subjective factors in data by introducing a smooth measure to state the place and role in the objects' class assignment. Given a set $U$, a fuzzy set $A$ is defined by means of its membership function $\mu_{A}: U \mapsto[0 . .1]$, such that, for any element $x \in U$, the membership value $\mu_{A}(x)$ is defined as: $\mu_{A}(x)=0$ if $x$ does not belong to $A, \mu_{A}(x)=1$ if $x$ belongs to $A$, whereas $0<\mu_{A}(x)<1$ if $x$ partially belongs to $A$.

A fuzzy atom is a formula $p\left(t_{1}, \ldots, t_{n}\right)$, where $t_{1}, \ldots, t_{n}$ are terms and $p$ is a predicate symbol. Fuzzy predicates can be regarded as fuzzy sets of tuples, that is $\mu\left(p\left(t_{1}, \ldots, t_{n}\right)\right)=\mu_{p}\left(t_{1}, \ldots, t_{n}\right)$, where $\mu_{p}$ is the membership function of predicate $p$. Given an atom $a$, the truth value $\mu(a)$ ranges in [0..1], and a fuzzy fact is an expression of the form $a \leftarrow \mu(a)$. In our setting, we mainly use built-in predicates, where the truth values of the ground atoms are pre-assigned. Truth value of conjunction and disjunction of atoms can be straightforwardly defined by means of aggregation operators 10. Formally, given two fuzzy atoms $p\left(t_{1}, \ldots, t_{n}\right)$ and $q\left(t_{1}^{\prime}, \ldots, t_{k}^{\prime}\right)$, we have:

$-\mu\left(p\left(t_{1}, \ldots, t_{n}\right) \wedge q\left(t_{1}^{\prime}, \ldots, t_{k}^{\prime}\right)\right)=\min \left(\mu\left(p\left(t_{1}, \ldots, t_{n}\right)\right), \mu\left(q\left(t_{1}^{\prime}, \ldots, t_{k}^{\prime}\right)\right)\right)$,

$-\mu\left(p\left(t_{1}, \ldots, t_{n}\right) \vee q\left(t_{1}^{\prime}, \ldots, t_{k}^{\prime}\right)\right)=\max \left(\mu\left(p\left(t_{1}, \ldots, t_{n}\right)\right), \mu\left(q\left(t_{1}^{\prime}, \ldots, t_{k}^{\prime}\right)\right)\right)$,

$-\mu\left(\neg p\left(t_{1}, \ldots, t_{n}\right)\right)=1-\mu\left(p\left(t_{1}, \ldots, t_{n}\right)\right)$.

As we shall explain in the following, fuzzy formulas enable modelling uncertainty in the wrapping process.

\subsection{Spatial Documents}

We refer to the concept of token as the basic element of a PDF document. A token is an atomic object (i.e. a textual element or an image), which is totally contained within a document page. The graphical representation of a token on the page layout takes up a certain room delimited by the token bounding box. We assume the presence of an alphabet $\Gamma$ of token values.

Definition 1 (Document token). A document token is a tuple $\left\langle v, p\right.$, inf $f_{x}$, inf $\sup _{x}$, sup $\left._{y}\right\rangle$, where $v \in \Gamma, p$ is a page number, inf $f_{x}$ and inf $f_{y}$ (resp., sup and $_{x}$ ap $_{y}$ ) are the coordinates (pixels) of the top-left corner (resp., bottom-right corner) of the token bounding box. A spatial document is a set of document tokens.

Reasoning about tokens and their relationships lies on the capability of defining suitable predicates to check syntactic as well as semantic properties, and to characterize the spatial properties between pairs of tokens. 
Spatial predicates allow for capturing relationships between locations of tokens. We denote with spatialrelation $\left(t_{1}, t_{2}\right)$ a type of predicate that holds if there exists a specific spatial relation between tokens $t_{1}$ and $t_{2}$. More precisely, spatial predicates include cardinal direction predicates, namely east, west, north, northeast, northwest, south, southeast, southwest, and topological predicates such as precedes and follows.

Cardinal direction predicates are defined as fuzzy predicates, whose truth values depend on spatial relationships between tokens. For instance, the truth value of an atom north $\left(t_{1}, t_{2}\right)$, defined on tokens $t_{1}$ and $t_{2}$, depends on the amplitude of the angle formed by a line connecting the center of $t_{1}$ to the center of $t_{2}$ and the vertical axis of the document. By contrast, topological predicates are more simple since we assume they admit only true or false as truth value.

Content predicates are defined on the content of tokens. Some useful content predicates are listed below:

- contains $\operatorname{Str}(t, s)$ : holds if string $s$ is contained in the text of token $t$;

- is Number $(t)$ : holds if token $t$ represents a number;

- value $(t, s)$ : holds if string $s$ is the text value of token $t$;

- regExp $(t, e)$ : holds if the text of token $t$ matches a regular expression $e$;

- concept $(t, c)$ : measures the relevance of token $t$ with respect to an ontology concept $c$.

In the following section, we shall give evidence that spatial and content predicates are the basis for setting constraints on the construction of token groups.

\section{Wrapping PDF Documents}

\subsection{PDF Extracted Information Model}

In order to extract desired information, individual tokens are hierarchically organized into groups. A token group collects logically related tokens. For instance, in Fig. 1. tokens appearing in a line of a balance sheet are to be grouped together to compose a balance item. Balance items of type Current Assets, Cash, T-bills, Accounts Receivable, and Total Current Assets are then grouped together to compose the current asset group and so on. We assume the presence of a finite alphabet $\mathcal{T}$ of group types.

Definition 2 (Token group). A token group is a pair $\langle\tau, \gamma\rangle$, where $\tau \in \mathcal{T}$ is a group type and $\gamma$ is either a sequence $\gamma=\left[\left\langle\tau_{1}, \gamma_{1}\right\rangle, \ldots,\left\langle\tau_{n}, \gamma_{n}\right\rangle\right]$ of token groups, or a single token.

Given a token group $g=\langle\tau, \gamma\rangle$, we denote with children $(g)$ the set of the groups appearing in $\gamma$, and with subgroups $(g)$ the set of the groups that either appear in $\gamma$ or are recursively contained in subgroups $\left(g^{\prime}\right)$ such that $g^{\prime} \in \gamma$.

As stated in Definition 2, each token group is associated with a group type, and may consist of more subgroups each having a specific type. Thus, a compound token group is characterized in terms of the group types corresponding to its subgroups. 
Definition 3 (Group content type). Given a token group $g=\langle\tau, \gamma\rangle$, the content type $\operatorname{cnt}(g)$ of $g$ is either $\tau_{1}, \ldots, \tau_{n}$ if $\gamma=\left[\left\langle\tau_{1}, \gamma_{1}\right\rangle, \ldots,\left\langle\tau_{n}, \gamma_{n}\right\rangle\right]$, or symbol $\epsilon \notin \mathcal{T}$ if $\gamma$ is a single token.

However, not all the token groups are well-suited to be considered as a result of the extraction task. In particular, we only consider "non-overlapping" groups, that is any group must not contain two identical subgroups. This must be true not only for the children of a group but also for all its descendants. For this purpose, the notion of well-formed group is next given.

Definition 4 (Well-formed group). A token group $g=\langle\tau, \gamma\rangle$ is said to be well-formed if and only if there not exist two groups $g^{\prime}, g^{\prime \prime} \in \operatorname{subgroups}(g)$, with $g^{\prime} \notin \operatorname{subgroups}\left(g^{\prime \prime}\right)$ and $g^{\prime \prime} \notin \operatorname{subgroups}\left(g^{\prime}\right)$, such that children $\left(g^{\prime}\right) \cap$ children $\left(g^{\prime \prime}\right) \neq \emptyset$.

Proposition 1. Let $g=\langle\tau, \gamma\rangle$ be a token group. If $g$ is well-formed, each $g^{\prime} \in \gamma$ is well-formed.

Let $g^{\prime}=\left\langle\tau^{\prime}, \gamma^{\prime}\right\rangle$ and $g^{\prime \prime}=\left\langle\tau^{\prime \prime}, \gamma^{\prime \prime}\right\rangle$ be two token groups, where $\gamma^{\prime}=\left[g_{0}^{\prime}, \ldots, g_{n}^{\prime}\right]$ and $\gamma^{\prime \prime}=\left[g_{0}^{\prime \prime}, \ldots, g_{m}^{\prime \prime}\right]$. We say that $g^{\prime}$ contains $g^{\prime \prime}\left(g^{\prime} \supseteq g^{\prime \prime}\right)$ if and only if:

$-g^{\prime}=g^{\prime \prime}$, or

- $n=m$ and, for each $i \in[0 . . n], g_{i}^{\prime} \supseteq g_{i}^{\prime \prime}$, or

$-n>m$ and there exists a sequence of indexes $i_{0}, \ldots, i_{m}$ such that, for each $j \in[0 . . m], i_{j}<i_{j+1}$ and $g_{i_{j}}^{\prime} \supseteq g_{j}^{\prime \prime}$.

\subsection{PDF Wrappers}

In order to define a PDF wrapper we have to specify how to create a group starting from previously recognized subgroups, and which conditions these subgroups must satisfy. However, the print-oriented nature of PDF documents makes it impossible to specify tight conditions. To overcome this limitation we possibly exploit fuzzy conditions on token groupings.

Spatial and content predicates, introduced in Section 2.2 for characterizing properties and relationships between tokens, can be easily extended for token groups. The underlying idea is that a content predicate holds for a group if it holds for all the token within the group. Analogously, a spatial relationship between two groups holds if it holds for all the pairs of tokens within the groups. For instance, given two groups $g_{1}$ and $g_{2}$, relation north $\left(g_{1}, g_{2}\right)$ can be formalized as follows: $\operatorname{north}\left(g_{1}, g_{2}\right) \rightarrow \forall t_{i} \in \gamma_{1}, t_{j} \in \gamma_{2}, \operatorname{north}\left(t_{i}, t_{j}\right)$. This guarantees the monotonicity property for cardinal direction predicates. Extension of topological predicates to token groups can be made straightforwardly, since such predicates are not defined by means of fuzzy constraints.

A fuzzy constraint is a disjunction of conjunctions of content and spatial predicates. Formally, given a set of variables $\mathcal{V}$, a fuzzy constraint $c(\mathcal{V})$ is a formula $\bigvee_{i=0}^{n} c_{i}\left(\mathcal{V}_{i}\right)$, where each $c_{i}\left(\mathcal{V}_{i}\right)$ is a conjunction of group atoms $\bigwedge_{j=0}^{k_{i}} a_{i, j}\left(\mathcal{V}_{i, j}\right)$ such that $\mathcal{V}_{i, j}$ is the set of all variables appearing in $a_{i, j}, \mathcal{V}_{i}=\bigcup_{j=0}^{k_{i}} \mathcal{V}_{i, j}$, and $\mathcal{V}=\bigcup_{i=0}^{n} \mathcal{V}_{i}$ 
Let $\mathcal{V}$ and $G$ be a set of variables and a set of groups, respectively, and let $\theta$ be a set of pairs $x / g$ such that $x \in \mathcal{V}$ and $g \in G$. $\theta$ is a group variable substitution if and only if it does not contain two pairs $x / g^{\prime}$ and $x / g^{\prime \prime}$ such that $g^{\prime} \neq g^{\prime \prime}$. Moreover, given a conjunction $c(\mathcal{V})$ of group atoms, a group variable substitution $\theta$ is said ground for $c(\mathcal{V})$ if and only if it contains a pair $x / g$ for each $x \in \mathcal{V}$.

As is usual in standard logic programming, the application of a substitution $\theta$ to a variable $x$ returns a group $g$ if $x / g \in \theta$, or $x$ itself otherwise. The result of the application of a substitution $\theta$ to an atom $a\left(x_{0}, \ldots, x_{l}\right)$, denoted with $\theta \circ a\left(x_{0}, \ldots, x_{l}\right)$, is an atom $a\left(\theta x_{0}, \ldots, \theta x_{l}\right)$. The application of a substitution to conjunction and disjunction of atoms is defined accordingly.

Proposition 2. Let $c$ be a conjunction of group atoms, and $\theta, \theta^{\prime}$ be ground group variable substitutions for $c$. If, for each $x / g \in \theta$, there is a pair $x / g^{\prime} \in \theta^{\prime}$ such that $g \subseteq g^{\prime}$ then $\mu(\theta \circ c) \geq \mu\left(\theta^{\prime} \circ c\right)$.

Group Type Definitions. The specification of group content model requires the conditions, which selected subgroups must satisfy, are defined with respect to the type of a group.

Given an alphabet $\mathcal{T}$ of group types and an alphabet $\mathcal{V}$ of variables, an annotated element name is defined as a pair $\tau: x$, such that $\tau \in \mathcal{T}$ and $x \in \mathcal{V}$. An annotated content model on $(\mathcal{T}, \mathcal{V})$ is either a one-unambiguous regular expression [1] on annotated element names, or a pair \#TOKEN : x, where \#TOKEN is the token content model and denotes a simple textual token 1

Given a group $g=\langle\tau, \gamma\rangle$ and an annotated content model $e$, the content type $\operatorname{cnt}(g)$ is valid for $e$ if:

$-\operatorname{cnt}(g)=\epsilon$ and $e=\# T O K E N: x$, or

- $\operatorname{cnt}(g)=\tau_{1}, \ldots, \tau_{n} \in L(e)$, where $L(e)$ denotes the language generated by $e$.

Given a group $g=\langle\tau, \gamma\rangle$ and an annotated content model $e$ such that $\operatorname{cnt}(g)$ is valid for $e$, the variable binding of $\gamma$ with respect to $e$, denoted with $e(g)$, is a set of pairs $\left\{g_{1} / x_{1}, \ldots, g_{n} / x_{n}\right\}$ such that $x_{i}$ is the variable implicitly associated to $g_{i}$ parsing $\gamma=\left[g_{1}, \ldots, g_{n}\right]$ with $e$. Moreover, we denote with $\Theta(e, g)$ the set of all the subsets of $e(g)$ that are group variable substitutions. Notice that, in general $e(g)$ is not a group variable substitution, since a variable can be associated to multiple subgroups.

Definition 5 (Group type definition). Let $\tau$ be a group type, e be an annotated content model on $(\mathcal{T}, \mathcal{V})$, and $c$ be a fuzzy constraint on $e$. A group type definition is a tuple $\langle\tau, e, c\rangle$.

\footnotetext{
${ }^{1}$ The "one-unambiguous" property for a regular expression allows for determining uniquely which position of a symbol in the expression should match a symbol in an input word, without looking beyond that symbol in the input word. For this reason, it is worth emphasizing that there is only one way by which a string of group types can be "parsed" using an annotated content model, thus an annotated content model implicitly associates a variable $x_{i}$ to each subgroup $\left\langle\tau_{i}, \gamma_{i}\right\rangle$ in $\gamma$.
} 
Roughly speaking, a group type definition is a complete specification of the content model, together with an additional fuzzy constraint. Moreover, a group type definition $\langle\tau, e, c\rangle$ refers to the type $\tau$. In the following, we characterize the validity of a token group of type $\tau$ with respect to a group type definition referring to $\tau$.

Definition 6 (Group validity). Let $g=\langle\tau, \gamma\rangle$ be a token group and gtd = $\left\langle\tau^{\prime}, e, c\right\rangle$ be a group type definition. We say that $g$ is valid with respect to $g$ td if $\tau=\tau^{\prime}$ and $\operatorname{cnt}(g)$ is valid for $e$.

The syntactic validity of groups being extracted is not affected by the application of fuzzy constraints. The truth value of a token group can be computed by combining the truth values of its subgroups with the truth values of the fuzzy constraints according to the rules defined in Section 2.1. However, we have to define how a constraint $c$ is grounded by the application of a group variable substitution $\theta$. It may happen that applying $\theta$ to $c$ the resulting formula is not ground, i.e. $\theta \circ c$ still contains some variables. In this case, the semantics of constraint intuitively requires that the conjunction in which a variable still appears will not be considered. The following example explains the above intuition.

Example 1. In Fig. 1 consider a group $g$ whose description is as follows:

\begin{tabular}{|c|c|c|}
\hline group & type $(\tau)$ & content $(\gamma)$ \\
\hline \hline$g$ & Assets & {$\left[g_{1}, g_{2}\right]$} \\
\hline$g_{1}$ & Current_Assets & $t_{1}$ \\
\hline$g_{2}$ & Intangible_Assets & $t_{2}$ \\
\hline
\end{tabular}

where $g_{1}, g_{2}$ are token groups and $t_{1}, t_{2}$ are tokens. Group $g$ is associated with the group type definition $g t d=\langle\tau, e, c\rangle$ such that $\tau=$ Assets, $e=$ Current_Assets : $C A$, (Inventory : $I \mid$ Intangible_Assets : $I A$ ), and $c=$ follows $(C A, I)$. By parsing $g$ with $e$, we have $e(g)=\left\{g_{1} / C A, g_{2} / I A\right\}$, which equals the set $\Theta(e, g)$ since it is a group variable substitution. By applying the substitution to $c$ we obtain the non-ground constraint follows $\left(g_{1}, I\right)$. Thus, the truth value of $g$ is provided by the minimum between the truth values of its subgroups (i.e. $g_{1}, g_{2}$ ).

Given a fuzzy constraint $c=c_{0} \vee \ldots \vee c_{n}$ and a group variable substitution $\theta$, the grounded version of $\theta \circ c$ is defined as $\operatorname{ground}(\theta \circ c)=\operatorname{ground}\left(\theta \circ c_{0}\right) \vee \ldots \vee$ $\operatorname{ground}\left(\theta \circ c_{n}\right)$, where $\operatorname{ground}\left(\theta \circ c_{i}\right)$ is $\theta \circ c_{i}$ if $\theta \circ c_{i}$ is ground, false otherwise.

Given a group $g=\langle\tau, \gamma\rangle$ which is valid for a group type definition $g t d=$ $\left\langle\tau^{\prime}, e, c\right\rangle$, the truth value of $g$ with respect to $g t d$ is given by the truth value of the formula

$$
\mu_{g t d}(g)=\mu\left(\bigwedge_{g^{\prime} \in \gamma} \mu\left(g^{\prime}\right) \wedge \operatorname{ground}(\theta \circ c), \forall \theta \in \Theta(e, g)\right),
$$

where for each possible variable binding we compute its truth value with respect to all ground constraints $\theta \circ c$. Once computed the truth value of each conjunct, the overall value is obtained by using the min operator (cf. Section 2.1). Notice 
that, the truth value of the overall formula propagates starting from the token value. Thus, token truth values bound the overall value of the formula and, as usual in classic logic, if any conjunct evaluates to 0 the overall formula will evaluate to 0 too.

PDF Wrapper Specification and Semantics. Faced with the above definitions, we are now ready to provide the notion of PDF wrapper.

Definition 7 (PDF wrapper). A PDF wrapper is a tuple $W=\langle\tau, G\rangle$, where $\tau \in \mathcal{T}$ is the root group type and $G=\left\{\left\langle\tau_{0}, e_{0}, c_{0}\right\rangle, \ldots,\left\langle\tau_{n}, e_{n}, c_{n}\right\rangle\right\}$ is a set of group type definitions such that $\tau_{i} \neq \tau_{j}$, for each $i, j \in[0 . . n], i \neq j$.

Let $W=\langle\tau, G\rangle$ be a PDF wrapper and $\left\langle\tau_{1}, e_{1}, c_{1}\right\rangle,\left\langle\tau_{2}, e_{2}, c_{2}\right\rangle \in G$ be two group type definitions. We say that $\tau_{1}$ depends on $\tau_{2}$ if $\tau_{2}$ appears in $e_{1}$, or there exists a group type definition $\left\langle\tau_{3}, e_{3}, c_{3}\right\rangle$ such that $\tau_{1}$ depends on $\tau_{3}$ and $\tau_{3}$ depends on $\tau_{2}$. A PDF wrapper is said to be non-recursive if it does not contain two group types $\tau_{1}, \tau_{2}$ such that $\tau_{1}$ depends on $\tau_{2}$. In the following we consider only non-recursive PDF wrappers.

Given a wrapper $W=\left\langle\tau_{0}, G\right\rangle$ and a group type $\tau$, we denote with $W(\tau)$ the group type definition of $\tau$ in $G$.

Definition 8 (Valid group). Let $W=\left\langle\tau_{0}, G\right\rangle$ be a PDF wrapper and doc be a PDF document. A group $g=\langle\tau, \gamma\rangle$ on doc is valid for $W$ if and only if:

1. $g$ is well-formed, and

2. $\tau=\tau_{0}$, and

3. $g$ is valid with respect to $W(\tau)$, and

4. for each subgroup $g^{\prime}=\left\langle\tau^{\prime}, \gamma^{\prime}\right\rangle$ of $g, g^{\prime}$ is valid with respect to $W\left(\tau^{\prime}\right)$.

The set of all groups over doc valid for $W$ is denoted as $\mathcal{G}(W$, doc $)$.

Broadly speaking, a valid group is essentially a well-formed group of tokens, which conforms to the schema defined by a wrapper. The reliability of the extracted data that are contained in a group $g$ is substantially fuzzily measured by $\mu_{g t d}(g)$. However, since the truth value of a group depends both on its associated $g t d$ and the truth values of its subgroups, in the following we denote with $\mu_{W}(g)$ the truth value of a group according to the definition of a wrapper $W$.

Given a PDF wrapper $W$ and a document $d o c$, there can be several different groups that are valid with respect to $W$. However, not all such groups are desirable as results of the evaluation of $W$ on doc. The key-idea is to consider only groups that are "maximal", i.e. groups whose truth value is equal to or greater than a predefined threshold, and which are not contained inside other valid groups that meet the truth value requirement as well.

Definition 9 (Maximal group). Let $W=\left\langle\tau_{0}, G\right\rangle$ be a PDF wrapper, $t$ be a truth value threshold, and doc be a PDF document. A group $g \in \mathcal{G}(W$, doc $)$ is said to be maximal with respect to $t$ if and only if: 
$-\mu_{W}(g) \geq t$, and

- there not exists $g^{\prime} \in \mathcal{G}(W$, doc $)$ such that $g^{\prime} \supseteq g$ and $\mu_{W}\left(g^{\prime}\right) \geq t$.

The set of all maximal groups with respect to $t$ is denoted as $\mathcal{M}_{t}(W$, doc $)$.

Clearly, it is possible to consider only the maximal groups having the greatest truth value. However, this strategy may be computationally expensive; thus, we rather prefer to adopt a greedy approach for searching a maximal group with the greatest truth value.

\section{PDF Wrapper Evaluation}

In the proposed approach, the objective of evaluating a wrapper for a PDF document is to compute a maximal token group from that PDF document. We consider a restricted form of wrappers, called $\star$-free wrappers, which do not contain optionals or repeated subgroups in group definitions.

Definition 10 ( $\star$-free wrapper). Let $W=\left\langle\tau_{0}, G\right\rangle$ be a PDF wrapper. $W$ is said to be $\star$-free if and only if, for each group type definition $\langle\tau, e, c\rangle \in G$, e does not contain $*$ or ?.

Given a wrapper $W$, we denote with $W^{\star}$ the wrapper obtained by replacing each group type definition $\langle\tau, e, c\rangle$ in $W$ with $\left\langle\tau, e^{\star}, c\right\rangle$, where $e^{\star}$ is the annotated content model obtained from $e$ by removing each occurrence of symbols $*$ and ?. This operation allows us to consider the "kernel" of the content model defining $W$ : indeed, as can be easily observed, any expression in $L\left(e^{\star}\right)$ is also in $L(e)$, that is $L\left(e^{\star}\right) \subseteq L(e)$.

The search for maximal groups works by repeatedly trying to add new subgroups to existing groups. This is achieved by expanding optional parts of group definition which have not been previously considered.

Definition 11 (Group expansion). Let $W=\left\langle\tau_{0}, G\right\rangle$ be a PDF wrapper, doc be a PDF document, $g=\langle\tau, \gamma\rangle$ and $g^{\prime}=\left\langle\tau, \gamma^{\prime}\right\rangle$ be two groups from doc, and $G(\tau)$ be $\langle\tau, e, c\rangle$. We say that $g^{\prime}$ is an expansion of $g$ if and only if $g \subseteq g^{\prime}$ and each pair $g_{i} / x_{i} \in e(g)$ also belongs to $e\left(g^{\prime}\right)$.

Lemma 1. Let $W$ be a PDF wrapper, doc be a PDF document, and $g, g^{\prime}$ two groups from doc. If $g^{\prime}$ is an expansion of $g$ then $\mu_{W}(g) \geq \mu_{W}\left(g^{\prime}\right)$.

Lemma 2. Let $W$ be a PDF wrapper, doc be a PDF document, and $t$ be a truth value threshold. For each group $g$ in $\mathcal{M}_{t}(W$, doc $)$, there exists a group $g^{\prime}$ in $\mathcal{M}_{t}\left(W^{\star}\right.$, doc) such that $g$ is an expansion of $g^{\prime}$.

Lemma 3. Let $W$ be a PDF wrapper, doc be a PDF document, and t be a truth value threshold. $\mathcal{M}_{t}(W$, doc $)$ is empty if and only if $\mathcal{M}_{t}\left(W^{\star}\right.$,doc $)$ is empty.

Theorem 1. Let $W$ be a PDF wrapper, doc be a PDF document, and $t$ be a truth value threshold. Checking if $\mathcal{M}_{t}(W$, doc $)$ is empty can be done in polynomial time with respect to the number of tokens in doc. 
Theorem 2. Let $W$ be a PDF wrapper, doc be a PDF document, and $t$ be a truth value threshold. Checking whether a group $g$ is in $\mathcal{M}_{t}(W, d o c)$ is feasible in polynomial time with respect to the number of tokens in doc.

\subsection{A Fuzzy Algorithm for Extracting Maximal Token Groups}

In this section we describe a PDF wrapper evaluation algorithm designed to extract a maximal token group from a PDF document (Fig. 21). Given a PDF document $d o c$ and a wrapper $W$ for it, the maximal token group can be extracted according to the content models specified in $W$ and to a predefined truth value threshold.

Initially, all the elementary token groups (i.e. groups of type \#TOKEN) are extracted from the source document doc. These groups are simply computed by selecting all the tokens that satisfy the truth value threshold with respect to the associated constraints. Then, the $\star$-free wrapper $W^{\star}$ is applied to $d o c$ to extract all the $\star$-free token groups; among these, the token group with the maximum truth value is chosen and recursively "expanded" while the group being constructed satisfies the desired truth value threshold.

Expanding a token group consists substantially in adding some subgroups to its content, that is re-defining its original annotated content model including the content of other groups. For this purpose, a normal form for annotated content models is exploited.

Definition 12 (Normal form annotated content model). An annotated content model $e=\exp _{1}, \ldots, \exp _{n}$ is in disjunction-free normal form if and only if, for each $i \in[1 . . n], \exp _{i}$ is either an annotated element name $\tau: x$ or an expression of the form exp?, or exp*, where exp is an annotated content model.

Given a group $g$ and an annotated content model $e$ in disjunction-free normal form, $g$ is matched by $e$ if and only if $\operatorname{cnt}(g)$ is valid for $e^{\star}$. In order to compute the expansion of a group $g$, the annotated content model $e$ in disjunction-free normal form that matches $g$ is considered. Let $e=\exp _{1}, \ldots, \exp _{n}$ be an annotated content model in disjunction-free normal form. When parsing $g$ with $e$, each $g_{i}=\left\langle\tau_{i}, \gamma_{i}\right\rangle$, such that $\gamma_{i} \in \operatorname{cnt}(g)$, is associated to one subexpression of $e$ which corresponds to a group type; then, a subexpression of the form exp? or exp* is chosen, and a group sequence $g_{1}^{\prime}, \ldots, g_{k}^{\prime}$ valid for $\exp _{i}$ is found.

As an example, consider a group $g=\left\langle\tau,\left[\left\langle a, \gamma_{1}\right\rangle,\left\langle b, \gamma_{2}\right\rangle,\left\langle c, \gamma_{3}\right\rangle\right]\right\rangle$ and an annotated content model in disjunction-free normal form $e=(a: x 1, b: x 2,(b: x 3 \mid d:$ $x 4) *, c: x 5)$ which matches $g$. Parsing $g$ with $e$ will result in assigning $x 1$ to $\left\langle a, \gamma_{1}\right\rangle, x 2$ to $\left\langle b, \gamma_{2}\right\rangle$, and $x 5$ to $\left\langle c, \gamma_{3}\right\rangle . g$ can be expanded by selecting a new group of type $b$ or $d$, and computing the groups $g^{\prime}=\left\langle\tau,\left[\left\langle a, \gamma_{1}\right\rangle,\left\langle b, \gamma_{2}\right\rangle,\left\langle b, \gamma_{4}^{\prime}\right\rangle,\left\langle c, \gamma_{3}\right\rangle\right]\right\rangle$ or $g^{\prime \prime}=\left\langle\tau,\left[\left\langle a, \gamma_{1}\right\rangle,\left\langle b, \gamma_{2}\right\rangle,\left\langle d, \gamma_{4}^{\prime \prime}\right\rangle\left\langle c, \gamma_{3}\right\rangle\right]\right\rangle$, respectively. Besides groups $g^{\prime}$ and $g^{\prime \prime}$, two annotated content models in disjunction-free normal form that match $g^{\prime}$ and $g^{\prime \prime}$, respectively, can be derived and further used to find new group expansions. For example, with respect to group $g^{\prime}$, the annotated content model $e^{\prime}=(a: x 1, b: x 2, b: x 3,(b: x 3 \mid d: x 4) *, c: x 5)$, which is in disjunction-free normal form, can be derived. 


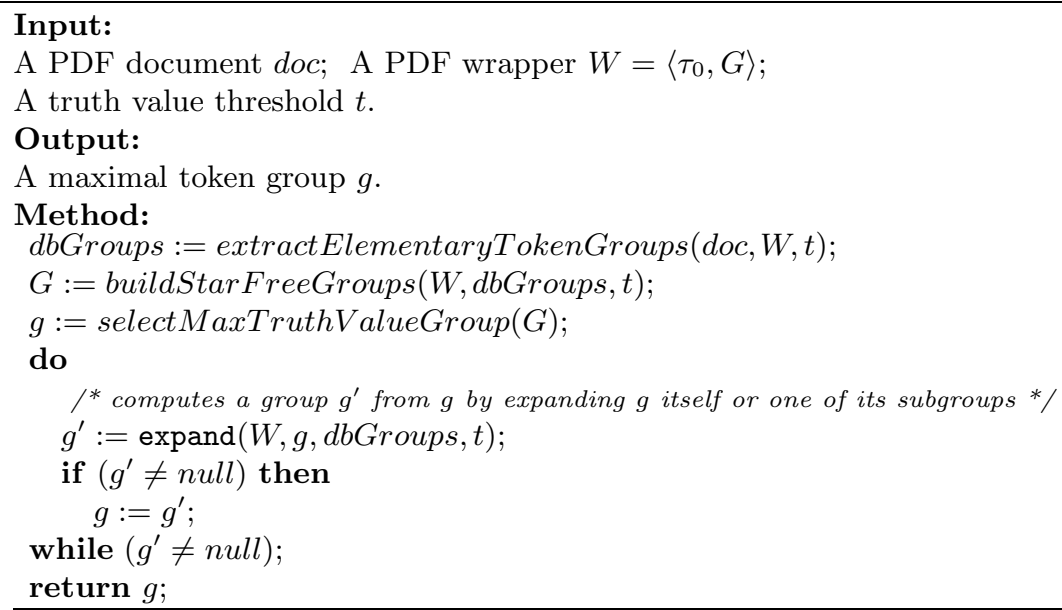

Function expand $(W, g, d b G r o u p s, t): g^{\prime}$;

\section{Method:}

$S:=\emptyset ; \quad g^{\prime}:=$ null;

for each $g^{\prime \prime}$ in descendantOrSelf $(g)$ do

Exp $:=$ findExpansion $\left(W, g^{\prime \prime}, d b G r o u p s, t\right)$;

for each $g g \in \operatorname{Exp}$ do

$g g^{\prime}:=\operatorname{replace}\left(g, g^{\prime \prime}, g g\right)$

if $g g^{\prime}$ is valid for $G\left(\tau_{0}\right)$ and $\mu_{W}\left(g g^{\prime}\right) \geq t$ and $g g^{\prime}$ is well-formed then $S:=S \cup\left\{g g^{\prime}\right\}$;

if $(S \neq \emptyset)$ then

$g^{\prime}:=$ selectMaxTruthValueGroup $(S)$;

return $g^{\prime}$;

Function findExpansion $(W, g, d b G r o u p s, t): \operatorname{Exp}$;

Method:

let $g=\langle\tau, \gamma, e\rangle$, with $e=\exp _{1}, \ldots, \exp _{n}$;

$\operatorname{Exp}:=\emptyset$;

for each $\exp _{i}$ of the form exp? or exp* do

$S:=$ instantiate $\left(\exp _{i}\right)$;

for each $e x \in S$ do

$S G:=\operatorname{selectGroups}\left(e^{\star} x^{\star}, d b G r o u p s\right) ;$

for each $s g \in S G$ do

let $e x^{\prime}=\exp _{1}, \ldots, \exp _{i-1}, \operatorname{ex}, \exp _{i+1}, \ldots, \exp _{n}$;

if $\left\langle\tau\right.$, compose $\left.\left(e x^{\prime}, \gamma, s g\right)\right\rangle$ is valid for $G(\tau)$ and

$\mu_{W}\left(\left\langle\tau, \operatorname{compose}\left(e x^{\prime}, \gamma, s g\right)\right\rangle \geq t\right.$ and

$\left\langle\tau\right.$, compose $\left.\left(e x^{\prime}, \gamma, s g\right)\right\rangle$ is well-formed then

$\operatorname{Exp}:=\operatorname{Exp} \cup\left\langle\tau, \operatorname{compose}\left(e x^{\prime}, \gamma, s g\right), e x^{\prime}\right\rangle$;

return Exp;

Fig. 2. The TokenGroupExtractor algorithm 
Let $g$ be a group to be expanded, and $e=\exp _{1}, \ldots, \exp _{n}$ be its matched annotated content model in disjunction-free normal form. The expansion of $g$ can be computed by instantiating each expression $\exp _{i} \in e$, that is by computing a set of annotated content models (in disjunction-free normal form) derived from $\exp _{i}$ and such that they do not parse the empty string. We define a function instantiate as follows:

- instantiate $(\exp *)=$ instantiate $(\exp ), \exp *$;

- instantiate $($ exp? $)=$ instantiate $(\exp )$;

- instantiate $\left(\exp _{1} \mid \exp _{2}\right)=$ instantiate $\left(\exp _{1}\right) \cup$ instantiate $\left(\exp _{2}\right)$;

- instantiate $\left(\exp _{1}, \exp _{2}\right)=$ instantiate $\left(\exp _{1}\right)$, instantiate $\left(\exp _{2}\right)$;

- instantiate $(\tau: x)=\tau: x$.

The concatenation of two sets of annotated content models $E_{1}, E_{2}$ is the set of annotated content models $\left\{e_{1}, e_{2} \mid e_{1} \in E_{1} \wedge e_{2} \in E_{2}\right\}$. We say that an expression of the form exp? or exp* is an expandable expression.

Let $g=\langle\tau, \gamma\rangle$ be a group and $e=\exp _{1}, \ldots, \exp _{n}$ be the annotated content model in disjunction-free normal form that matches $g$. Let $\exp _{i}$ be an expandable expression, $e x_{i}$ be an expression in instantiate $\left(\exp _{i}\right), \gamma$ be $\left[g_{1}, \ldots, g_{n}\right]$, and $g_{1}^{\prime}, \ldots, g_{k}^{\prime}$ be a sequence of groups matching $e x_{i}$. The sequence $\left[g_{1}, \ldots, g_{j}, g_{1}^{\prime}, \ldots\right.$, $\left.g_{k}^{\prime}, g_{j+1}, \ldots, g_{n}\right]$ matching $e x=\exp _{1}, \ldots, \exp _{i-1}, \operatorname{ex}_{i}, \exp _{i+1}, \ldots, \exp _{n}$, is denoted as compose $\left(e x, \gamma,\left[g_{1}^{\prime}, \ldots, g_{k}^{\prime}\right]\right)$.

We are now able to gain an insight into the TokenGroupExtractor algorithm of Fig. 2. We assume that a database dbGroups is used to store extracted annotated groups. An annotated group is a triplet of the form $g=\langle\tau, \gamma, e\rangle$, where $\langle\tau, \gamma\rangle$ is a group and $e$ the annotated content model in disjunction-free normal form that matches $\langle\tau, \gamma\rangle$.

Once elementary token groups have been extracted and stored into dbGroups, function buildStarFreeGroups first normalizes the input wrapper by producing, for each $g t d \in G$, group type definitions in disjunction-free normal form; then, following the order derived from the dependence relation between group types, for each normalized group type definition $\langle\tau, e, c\rangle$ it computes $\star$-free groups by invoking selectGroups $\left(e^{\star}, d b G r o u p s\right)$, selects those that are well-formed and satisfy the desired truth value threshold, annotates them with the content model $e$, and finally adds the computed groups into $d b G r o u p s$.

Function expand tries to expand a group $g$ and returns an expanded group if possible, null otherwise. Among all possible ways of expanding $g$, function expand chooses the one exhibiting the highest truth value. Given three groups $g, g^{\prime}$, and $g g$, function replace returns a new group obtained by replacing $g^{\prime}$ with $g g$ inside $g$ if $g^{\prime}$ is a subgroup of $g$, or returns $g g$ otherwise. Moreover, function descendantOrSelf, given a group $g$, yields the set of all the subgroups of $g$ and $g$ itself. Finally, function selectMaxTruthValueGroup, given a set of groups $S$, returns the group with the maximum truth value in $S$.

Function findExpansion computes a set of all the possible expansions of an annotated group with respect to the wrapper, the group database, and the truth value threshold. Function selectGroups, given a $\star$-free annotated content model $e=\tau_{1}: x_{1}, \ldots, \tau_{n}: x_{n}$ (in disjunction-free normal form) and dbGroups, returns 
the set of group sequences of the form $\left\{g_{1}, \ldots, g_{n} \mid \forall i g_{i} \in \pi_{\tau_{i}}(d b G\right.$ roups $\left.)\right\}$, where $\pi_{\tau_{i}}(d b G$ roups $)$ is the set $\left\{g \mid g \in d b G r o u p s \wedge\right.$ type $\left.(g)=\tau_{i}\right\}$.

Theorem 3. Let $W$ be a PDF wrapper, doc be a PDF document, and $t$ be a truth value threshold. If $\mathcal{M}_{t}(W$, doc $)$ is not empty, the TokenGroupExtractor algorithm computes a maximal group $g$ in $\mathcal{M}_{t}(W$, doc $)$ in polynomial time with respect to the number of tokens in doc.

\section{A Case Study: Wrapping Balance Sheets}

To assess the effectiveness of the PDF wrapping framework, we considered a collection of balance sheets made publicly available from Italian companies; it is highly heterogeneous due to the variety of formatting styles used to report the balance assets and liabilities. For the sake of brevity, we describe a significant example of information extraction from a page of the test balance sheet shown in Fig. 3 .

Suppose we would like to extract items each containing a balance voice (i.e. the item label) and two currency values referring to different fiscal years. Table 1 summarizes details about the specification of a wrapper suitable to the example balance sheet.

The maximal group to be extracted is of type item_collection. This group is composed of token groups item, which in turn consist of triplets of type (balance_voice, amount, amount). Each group item is constrained by a conjunction of two cardinal direction predicates. Once a group item has been built,

\begin{tabular}{|c|c|c|}
\hline STATO PATRIMONIALE - ATTIVO & $31 / 12 / 2003$ & $31 / 12 / 2002$ \\
\hline \multicolumn{3}{|l|}{ B) IMMOBILIZZAZIONI } \\
\hline \multicolumn{3}{|l|}{ I) IMMOBILIZZAZIONI IMMATERIALI } \\
\hline 1) Costi di impianto e di ampliamento & 10.739 & 73.792 \\
\hline 5) Avviamento & 433.824 & 495.799 \\
\hline I TOTALE IMMOBILIZZAZIONI IMMATERIALI & 444.563 & 569.591 \\
\hline \multicolumn{3}{|l|}{ II) IMMOBILIZZAZIONI MATERIALI } \\
\hline \multicolumn{3}{|l|}{ 2) Impianti e macchinari } \\
\hline a) impianti e macchinari & 399.839 & 336.282 \\
\hline b) fa.impianti e macchinari & 169.253- & $105.762-$ \\
\hline 2 TOTALE Impianti e macchinari & 230.586 & 230.520 \\
\hline \multicolumn{3}{|l|}{ 3) Attrezzature industriali e commerciali } \\
\hline a) attrezzature industriali e commerciali & 63.045 & 61.845 \\
\hline b) fa.attrezzature industriali e commerciali & 47.446- & $29.561-$ \\
\hline 3 TOTALE Attrezzature industriali e commerciali & 15.599 & 32.284 \\
\hline \multicolumn{3}{|l|}{ 4) Altri beni } \\
\hline a) altri beni & 19.693 & 18.703 \\
\hline b) fa.altri beni & $11.094-$ & $6.621-$ \\
\hline 4 TOTALE Altri beni & 8.599 & 12.082 \\
\hline II TOTALE IMMOBILIZZAZIONI MATERIALI & 254.784 & 274.886 \\
\hline
\end{tabular}

Fig. 3. Sample page of the test Italian company's balance sheet 
Table 1. A wrapper for the balance sheet of Fig. 3

\begin{tabular}{|l|l|}
\hline$W=\left\langle\tau_{0}, G\right\rangle$ & $\tau_{0}=$ item_collection \\
& $G=\left\langle g t d_{1}, g t d_{2}, g t d_{3}, g t d_{4}\right\rangle$ \\
\hline$g t d_{1}=\left\langle\tau_{1}, e_{1}, c_{1}\right\rangle$ & $\tau_{1}=\tau_{0}$ \\
& $e_{1}=($ item $: I T) *$ \\
& $c_{1}=$ True () \\
\hline$g t d_{2}=\left\langle\tau_{2}, e_{2}, c_{2}\right\rangle$ & $\tau_{2}=$ item \\
& $e_{2}=$ balance_voice $:$ BV, amount $: N_{1}$, amount $: N_{2}$ \\
& $c_{2}=$ west $\left(B V, N_{1}\right) \wedge$ west $\left(N_{1}, N_{2}\right)$ \\
\hline$g t d_{3}=\left\langle\tau_{3}, e_{3}, c_{3}\right\rangle$ & $\tau_{3}=$ balance_voice \\
& $e_{3}=\sharp T O K E N: X_{1}$ \\
& $c_{3}=$ concept $\left(X_{1}\right.$, balance_voice_object $)$ \\
\hline$g t d_{4}=\left\langle\tau_{4}, e_{4}, c_{4}\right\rangle$ & $\tau_{4}=$ amount \\
& $e_{4}=\sharp T O K E N: X_{2}$ \\
& $c_{4}=$ isNumber $\left(X_{2}\right)$ \\
\hline
\end{tabular}
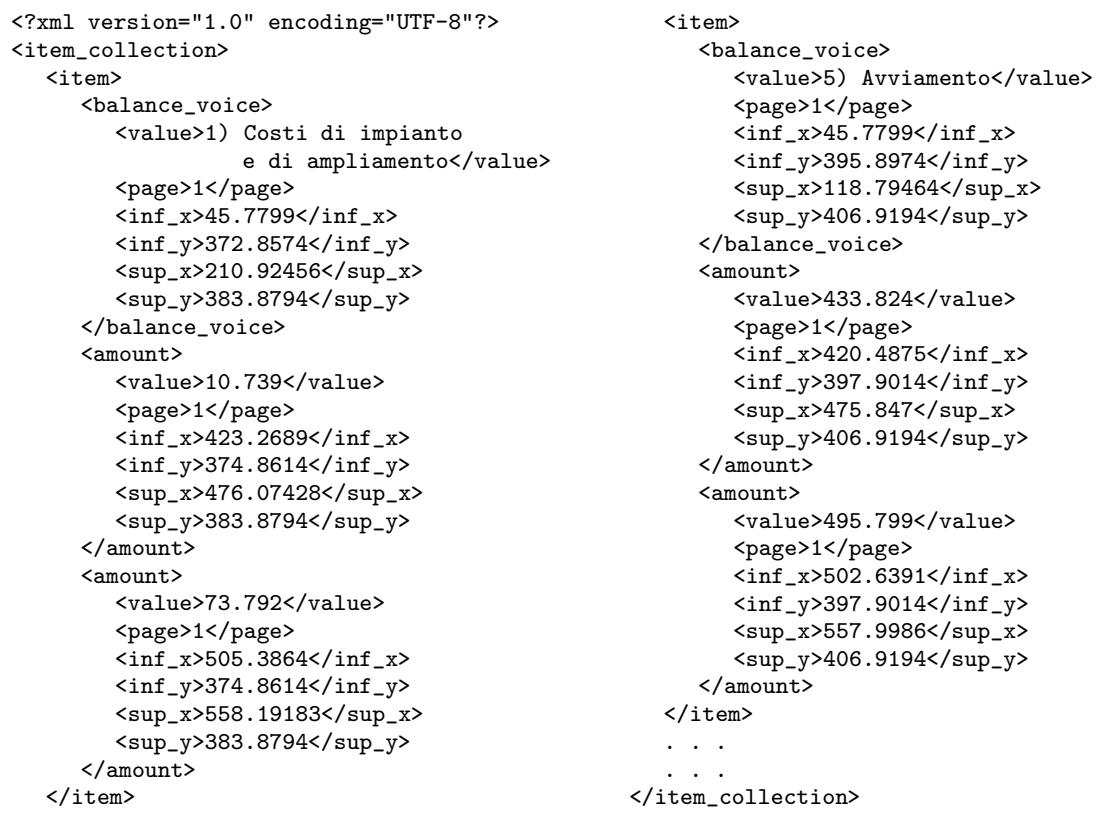

Fig. 4. XML document extracted from the balance sheet of Fig. 3

it is added to the content of the group of type item_collection, without being subject to a real constraint (i.e. constraint $c_{1}$ always holds).

Another noteworthy remark concerns $c_{3}$. This constraint checks whether a token associated to type balance_voice really represents a balance voice. To accomplish this, a predicate concept evaluates the membership of a given token as an instance of class balance_voice_object, according to some functions which 
compute at what degree a string (token value) conceptually matches a class property (e.g. a domain-specific cue phrase). Figure 4 shows an XML fragment representing the maximal token group of type item_collection extracted by evaluating the wrapper of Table 1] with a truth value threshold set to 0.8 .

\section{Conclusion}

We have presented a novel PDF wrapping framework based on a bottom-up approach, in which the extraction task consists in grouping together document tokens. A wrapper is defined by specifying the content for each type of token group. Annotated content models and fuzzy constraints as the basic elements of group type definitions. Fuzzy constraints are used to impose spatial and logical conditions on the content of each group. We have defined a declarative semantics of PDF wrappers and provided a polynomial time algorithm for extracting maximal groups. We have given evidence that fuzzy constraints are well-suited to capture subjective factors that brand the authorship in logically structuring information into a PDF document.

A system prototype is in advanced phase of development 2 and is currently being applied for extracting information from balance sheets.

\section{References}

1. Ashish, N., Knoblock, C.A.: Wrapper Generation for Semistructured Internet Sources. ACM SIGMOD Record 26(4) (1997) 8-15

2. Baumgartner, R., Flesca, S., Gottlob, G.: Visual Web Information Extraction with Lixto. In: Proc. VLDB '01 Conf. (2001) 119-128

3. Crescenzi, V., Mecca, G., Merialdo, P.: RoadRunner: Towards automatic data extraction from large Web sites. In: Proc. VLDB '01 Conf. (2001) 109-118

4. Freitag, D.: Machine Learning for Information Extraction in Informal Domains. Machine Learning 39(2-3) (2000) 233-272

5. Muslea, I., Minton, S., Knoblock, C.: Hierarchical Wrapper Induction for Semistructured Information Sources. Autonomous Agents and Multi-Agent Systems 4(1/2) (2001) 93-114

6. Soderland, S.: Learning Information Extraction Rules for Semistructured and Free Text. Machine Learning 34(1-3) (1999) 233-272

7. Laender, A., Ribeiro-Neto, B., da Silva, A., Teixeira, J.: A Brief Survey of Web Data Extraction Tools. ACM SIGMOD Record 31(2) (2002) 84-93

8. Adobe Systems Incorporated: PDF Reference, 5th edition: Adobe Portable Document Format version 1.6. Available at http://partners.adobe.com/public/ developer/pdf (2004)

9. Zadeh, L.: Fuzzy Sets. Information and Control 8 (1965) 338-353

10. Wygralak, M.: Fuzzy Cardinals based on the Generalized Equality of Fuzzy Subsets. Fuzzy Sets \& Systems 18 (1986) 143-158

11. Bruggemann-Klein, A., Wood, D.: One-Unambiguous Regular Languages. Information and Computation 142(2) (1998) 182-206

\footnotetext{
${ }^{2}$ http://www.deis.unical.it/tagarelli/pdf-wrapping
} 